\title{
Overnight Access to Sugar Solutions Affects mRNA Expression of Several Neuropeptides in Different Hypothalamic Regions in Rats
}

\author{
Changhui Zhao, Eric S. Campbell, Anna E. Tschiffely, Thomas W. Castonguay* \\ Department of Nutrition and Food Science, University of Maryland, College Park \\ *Corresponding author: twc@umd.edu
}

Received January 14, 2015; Revised January 24, 2015; Accepted February 01, 2015

\begin{abstract}
It has been known for years that free access to sugar solutions can cause weight gain and/or obesity in rats. We recently reported that brief access to sugar solutions can affect the hypothalamic neuropeptides that help to regulate energy balance. In this paper, we present the results in which we examined the effects of these sugars on the expression of several neuropeptides within specific hypothalamic regions. We provided Sprague Dawley rats $24 \mathrm{~h}$ access to $15 \%$ solutions of glucose, fructose, sucrose or high fructose corn syrup (HFCS) and then dissected portions of the paraventricular hypothalamic nuclei (PVN), the ventromedial hypothalamus (VMH) and the lateral hypothalamus (LH). We then evaluated the expression of several neuropeptides in these tissues, all of which were previously shown to be influenced by free access to sugar solutions using PCR array. Of the four sugar solutions tested, only fructose decreased expression of cholecystokinin (CCK) significantly, and only in the PVN. Glucose and sucrose significantly increased the expression of Tumor Necrosis Factor $\alpha$ (TNF- $\alpha$ ) only in the PVN. Fructose and sucrose decreased Growth Hormone $(\mathrm{GH})$ in the VMH. Further analysis indicated that it was fructose intake that was negatively correlated with both CCK and GH expression. Rats that had access to sugar solutions consumed less chow but maintained control levels of total caloric intake. We conclude that $24 \mathrm{~h}$ free access to different sugars can influence the expression of several hypothalamic neuropeptides in different ways. Changes in the expression of these neuropeptides do not disrupt total daily energy intake immediately but may nevertheless contribute to the obesity caused by long term access to sugar solutions.
\end{abstract}

Keywords: fructose, HFCS, glucose, sucrose, energy regulation

Cite This Article: Changhui Zhao, Eric S. Campbell, Anna E. Tschiffely, and Thomas W. Castonguay, "Overnight Access to Sugar Solutions Affects mRNA Expression of Several Neuropeptides in Different Hypothalamic Regions in Rats.” Journal of Food and Nutrition Research, vol. 3, no. 1 (2015): 69-76. doi: 10.12691/jfnr-3-1-12.

\section{Introduction}

It has been known for years that long term ad libitum access to sugar solutions (glucose, sucrose or fructose) can induce excessive weight gain and/or obesity in rats [1,2]. Similarly, long term free access to HFCS -55 solutions can also induce overweight and obesity [3]. When laboratory rats are fed a standard chow diet, they maintain energy balance: they match energy expenditure and energy intake [4]. Giving rats long term access to sugar solutions disrupts energy balance so as to induce obesity. The question is: "Which mechanism(s) is (are) involved in promoting sugar induced obesity?”

Energy intake is controlled by both peripheral and central regulators. Not all sugars are equally effective in promoting changes in these metabolic controls. For example, unlike glucose intake, fructose intake can quickly induce hypertriglyceridemia [5], a condition that frequently coexists with obesity, type 2 diabetes and metabolic syndrome. We have recently reported that fructose consumption can quickly suppress the expression of $11 \beta$-hydroxysteroid dehydrogenase type 1 (11 $\beta$-HSD1) in liver and visceral adipose tissue [6] leading to regeneration of intracellular glucocorticoids. Increased intracellular glucocorticoids are commonly observed in obesity in human and animal models $[7,8]$. Both of these effects are examples of how a nutrient (in this case sugars) can have a dramatic effect on gene expression and in that way change short term regulation.

Sugars can have differing effects on the hypothalamus, the brain's food intake control center, where appetite, motivation and reward processing functions are regulated [9]. Different hypothalamic structures and regions influence hunger and satiety. For example, Stellar proposed that the VMH and LH acted together to control food intake [10]. The "Dual Center Hypothesis" was one of the most studied theses in $20^{\text {th }}$ century neurophysiology. The PVN was added to this mix later, noting that there were differences in metabolic and behavioral controls of hunger [11]. Many neuropeptides synthesized in these hypothalamic regions play critical roles in energy maintenance [4]. 
We have hypothesized that brief access to sugar solutions can change the expression pattern of specific hypothalamic neuropeptides that control energy balance. We recently measured 84 obesity-related genes (using PCR arrays) in the hypothalamus of Sprague Dawley rats fed different sugar solutions. We found that several hypothalamic neuropeptides are affected differently by different sugar intakes from solution [12]. These neuropeptides include CCK, TNF- $\alpha$, GH, receptor activity modifying peptide (RAMP3), corticotropin releasing hormone (CRH) and thyrotropin releasing hormone (TRH). Interestingly, two important orexigenic neuropeptides -neuropeptide Y (NPY) and Agouti related peptide (AgRP) in the arcuate nucleus (ARC) were not changed after sugar access. It might be that brief sugar intake from solution somehow bypasses ARC and acts on the other hypothalamic regions. As a result, the purpose of the present study was to examine how these neuropeptides were affected by different sugars in three hypothalamic regions - the PVN, the $\mathrm{VMH}$ and the $\mathrm{LH}$.

\section{Materials and Methods}

\subsection{Animals}

Forty adult male Sprague-Dawley (CD strain) rats of approximately $300 \mathrm{~g}$ body weight (Charles River Laboratories, Wilmington, MA) were used. They were maintained on a $12 \mathrm{~h}$ light/dark cycle (lights on at 0800h) in a temperature controlled animal room $\left(22 \pm 1^{\circ} \mathrm{C}\right)$. During a 1 week acclimation period the rats were given free access to water and chow. The chow was a nutritionally complete low fat diet [Rodent diet 7012] prepared by Harlan Teklad (Bethlehem, PA). The chow contained $3.41 \mathrm{kcal} / \mathrm{g}$ of diet, of which $2.14 \mathrm{kcals}$ were derived from carbohydrate, 0.79 kcals were derived from protein, and 0.51 kcals derived from fat. All animals were given free access to chow and water throughout the experiment.

\subsection{Sugar Treatment}

Rats were randomly assigned to one of five weightmatched groups ( $n=8$ /group). One group of rats had ad libitum chow and water and served as the control group. Rats assigned to the other groups had ad libitum access to chow and water as well as to one of four solutions: a 15\% weight/volume (w/v) fructose (Tate \& Lyle, Decatur IL) solution, a 15\% (w/v) glucose (Sigma Aldrich, St Louis MO) solution, a $15 \%$ (total solute per volume) high fructose corn syrup (HFCS) (IsoSweet ${ }^{\circledR}$ 5500, 55\% fructose $-41 \%$ glucose, $77 \%$ solids, Tate \& Lyle, Decatur IL) or a $15 \%(w / v)$ sucrose (Domino Foods, Baltimore MD) solution. All sugar solutions were prepared $24 \mathrm{~h}$ in advance and stored at $4^{\circ} \mathrm{C}$ until used. The rats were maintained with free access to their respective diets for $24 \mathrm{~h}$ before sacrifice (at approximately 9:00 am). This method was chosen so as to minimize the stress associated with administering fixed volumes of solution intragastrically, as weight gain is thought to be facilitated by increased glucocorticoids. All rats were killed by slow replacement of air in a specialized chamber with pure $\mathrm{CO}_{2}$ followed by rapid decapitation and exsanguination. At the time of sacrifice, the brains were dissected, snap frozen in isopentane/dry ice and then stored at $-80{ }^{\circ} \mathrm{C}$ until use.

\subsection{Brain Sectioning}

Frozen brains were embedded using M1 embedding matrix (Lipshaw, Pittsburgh, PA) on dry ice. An IEC Minot Custom Microtome (Damon/IEC Division) was used for cryosectioning. The cryostat's blade and antiroll plate were pretreated with RNaseZap ${ }^{\circledR}$ to remove any possibility of RNase contamination and then cleaned with a paper towel soaked in DEPC-treated water. Slices were obtained from Interaural $7.70 \mathrm{~mm}$ (Bregma $-1.30 \mathrm{~mm}$ ) to Interaural $4.48 \mathrm{~mm}$ (Bregma $-4.52 \mathrm{~mm}$ ) at a thickness of $110 \mu \mathrm{m}$ each and carefully transferred to pre-cleaned slides (Fisher Scientific, Pittsburgh, PA). The slices were then stored at $-80^{\circ} \mathrm{C}$ until sampled.

\subsection{Sampling of Hypothalamic Regions}

RNA is unstable and subject to degradation. When following standard staining procedures, brain slices can lose as much as $10 \%$ of their initial RNA in as little as 30 minutes [13]. Water is the key factor that facilitates RNA degradation, however RNA integrity can be preserved up to $90 \mathrm{~min}$ following ethanol dehydration [13]. Preliminary tests showed that cresyl violet staining did not reveal internal hypothalamic structures quickly. Rather than attempt to stain the sections, we employed a three-step alcohol dehydration procedure (95\% alcohol immersion for 30 seconds, followed by $100 \%$ alcohol immersion for $1 \mathrm{~min}$ and then another $100 \%$ alcohol immersion for at least an additional minute). After dehydration, the slices were immediately dissected under a light microscope. Centered on the third ventricle, several $110 \mu \mathrm{m}$ slices of each region (PVN, VMH and $\mathrm{LH}$ ) were dissected using sterile 23 G x 1" hypodermic needles (B-D PrecisionGlide, Franklin Lakes. NJ). Specifically, 6-7 brain slices were used for PVN dissection. PVN sampling was initiated approximately at $-1.30 \mathrm{~mm}$ behind Bregma. VMH and LH samples were dissected starting at around $-2.12 \mathrm{~mm}$ behind Bregma. Captured tissues were carefully transferred into $1.5 \mathrm{ml}$ polypropylene eppendorf tubes on ice. $350 \mu \mathrm{l}$ RLT lysis buffer (containing10 $\mu \mathrm{l} \beta$ mercaptoethanol per $1 \mathrm{ml}$ Buffer RLT) was added and samples were then subjected to $30 \mathrm{sec}$ vortexing for cell breakage and RNA release. Samples were stored at $-80^{\circ} \mathrm{C}$ until RNA extraction.

\subsection{RNA Extraction and cDNA Synthesis}

Tissue lysates were thawed in a $37^{\circ} \mathrm{C}$ water bath until all the salts were dissolved. $\mathrm{VMH}$ and $\mathrm{LH}$ lysates were centrifuged using an Eppendorf centrifuge (model 5424) at full speed for $3 \mathrm{~min}$ to remove the cell debris. Samples from all three regions were then processed using a Qiagen RNeasy micro kit. This kit included DNase I to remove DNA that might affect the downstream applications. RNA quality was examined using a NanoDrop 2000 spectrophotometer (A260/A280 >1.8). The cDNA synthesis was completed using iScript ${ }^{\mathrm{TM}}$ cDNA Synthesis Kit (Bio-Rad) following the manufacturer's protocol. The final cDNA products were stored at $-20^{\circ} \mathrm{C}$ until use. 


\subsection{Quantitative real time PCR}

PCR reactions were performed in two replicates using iQ SYBR Green Supermix and a Bio-Rad CFX96 BioRad system. The program used for all PCR reactions was $95^{\circ} \mathrm{C}$ for $3 \mathrm{~min}$ and 40 cycles of $95^{\circ} \mathrm{C}$ for $15 \mathrm{sec}$, annealing temperature (Ta) for 30 sec (see Table 1) and $68^{\circ} \mathrm{C}$ for 30 sec. A melting curve program was then appended. All primers that were designed using Beacon Designer 7 software met the $\Delta \Delta \mathrm{Ct}$ requirement for the product length less than $200 \mathrm{bp}$.

Table 1. Primer set

\begin{tabular}{|c|c|c|c|}
\hline Primer Name & sequences (5' to 3') & Product Length (bp) & Ta $\left({ }^{\circ} \mathrm{C}\right)$ \\
\hline RPLP1 sense & GAAGAATCCGAGGATGACA & \multirow{2}{*}{81} & \multirow{2}{*}{51} \\
\hline RPLP1 antisense & CAGGTTCAGCTCTTTATTGG & & \\
\hline CCK sense & GCGTTTATTTATTAAGTCC & \multirow{2}{*}{133} & \multirow{2}{*}{43} \\
\hline CCK antisense & ATAGCATAGCAACATTAG & & \\
\hline Tnf- $\alpha$ sense & CCAATCTGTGTCCTTCTAA & \multirow{2}{*}{85} & \multirow{2}{*}{47} \\
\hline Tnf- $\alpha$ antisense & TTCTGAGCATCGTAGTTG & & \\
\hline RAMP3 sense & CAAGGTCATCTGGAAGGT & \multirow{2}{*}{109} & \multirow{2}{*}{50} \\
\hline RAMP3 antisense & GACTCСТААСААСТССАТТС & & \\
\hline GH sense & GTCTGTTTGCCAATGCTGTG & \multirow{2}{*}{152} & \multirow{2}{*}{55} \\
\hline GH antisense & TGGGATGGTCTCTGAGAAGC & & \\
\hline TRH sense & AAAGACATTGAAGCTGAAGAGAGG & \multirow{2}{*}{75} & \multirow{2}{*}{55} \\
\hline TRH antisense & GGGGTGCTGTCGTTTGTG & & \\
\hline CRH sense & TGGAGATTATCGGGAAAT & \multirow{2}{*}{158} & \multirow{2}{*}{47} \\
\hline CRH antisense & TACATCTTCTATGCTTCAAG & & \\
\hline
\end{tabular}

\subsection{Data Analysis}

The formula below was used to plot the final result from qPCR data:

$$
\begin{gathered}
\Delta C t(\text { test })=C t(\text { target,test })-C t(\text { ref }, \text { test }) \\
\Delta \mathrm{Ct}(\text { calibrator }) \\
=\mathrm{Ct}(\text { target, calibrator })-\mathrm{Ct}(\text { ref }, \text { calibrator }) \\
2^{-\Delta \Delta \mathrm{Ct}}=2^{\Delta \mathrm{Ct}(\text { calibrator })-\Delta \mathrm{Ct}(\text { test })}
\end{gathered}
$$

The Ct mean value from ribosomal protein, large, P1 (RPLP1) was used as the reference gene as before [12] and the water treated group mean $\mathrm{Ct}$ was used as the calibrator. All values were expressed as means \pm SEM. One way ANOVA with Duncan post hoc testing was applied to food intake and energy intake using IBM SPSS Statistics 21. Student's t tests were used to determine significance between groups using JMP Pro 10.0.2.
Pearson's correlation coefficients were calculated to evaluate the relationship between sugar intake from solution and the expression of the specific neuropeptides. $\mathrm{P}$ value less than 0.05 was considered statistically significant.

\section{Results}

\subsection{Food and Energy Intake}

When sugars were presented, the chow intake in all sugar fed groups decreased significantly compared with the control group. Although rats fed with sucrose and HFCS consumed nearly 10\% more energy compared with the control, the total caloric intakes of each group over the $24 \mathrm{~h}$ experimental period did not significantly differ from one another. The percentage of total calories derived from sugar intake from solution ranged from 44 to 53\%. See Table 2 for details.

Table 2. Calorie intake of rats fed with different sugars

\begin{tabular}{|l|l|l|l|l|l|}
\hline Group & Water & Glucose & Sucrose & HFCS & Fructose \\
\hline Chow Intake (g) & $28.8 \pm 3.3^{\mathrm{a}}$ & $14.1 \pm 1.6^{\mathrm{b}}$ & $15.2 \pm 2.3^{\mathrm{b}}$ & $17.5 \pm 2.7^{\mathrm{b}}$ & $17.6 \pm 3.7^{\mathrm{b}}$ \\
\hline Sugar Intake from Solution (g) & NA & $11.5 \pm 0.6$ & $13.2 \pm 1.0$ & $11.1 \pm 0.9$ & $9.7 \pm 1.4$ \\
\hline Chow Calorie (Kcal) & $93.1 \pm 10.8^{\mathrm{a}}$ & $46.2 \pm 5.2^{\mathrm{b}}$ & $49.56 \pm 7.4^{\mathrm{b}}$ & $57.18 \pm 8.7^{\mathrm{b}}$ & $57.67 \pm 12.7^{\mathrm{b}}$ \\
\hline Sugar Calorie from Solution (Kcal) & NA & $46.2 \pm 2.2$ & $52.71 \pm 3.9$ & $44.6 \pm 3.4$ & $38.8 \pm 5.7$ \\
\hline Total Calorie (Kcal) & $93.1 \pm 10.8$ & $92.3 \pm 4.8$ & $102.3 \pm 7.8$ & $101.8 \pm 7.0$ & $96.4 \pm 15.2$ \\
\hline \% calories intake as sugar from solution & NA & $51.1 \pm 4.3$ & $52.8 \pm 4.9$ & $45.9 \pm 5.6$ & $44.3 \pm 5.2$ \\
\hline
\end{tabular}

Note: Values are means \pm SEM

Values sharing a common superscript are not different from one another $(\mathrm{P}>0.05)$.

\subsection{Neuropeptides Regulated by Sugars}

\subsubsection{CCK}

Fructose downregulated CCK expression in the PVN $(p<0.05$; Figure 1A). CCK expression was not changed in the VMH or the LH (Figure 1B and 1C).

\subsubsection{TNF- $\alpha$}

Glucose and sucrose intake significantly increased the expression of TNF- $\alpha$ in the PVN $(\mathrm{P}<0.05)$. HFCS and fructose groups failed to change TNF- $\alpha$ in the PVN (Figure 1D). No differences between any groups were found in the VMH or the LH (Figure 1E and 1F). 


\subsubsection{GH}

We failed to detect any GH in the PVN. In the VMH, sucrose and fructose decreased GH expression (Figure 1G).
In the LH, sucrose and fructose decreased GH expression when compared with glucose (Figure $1 \mathrm{H}$ ).
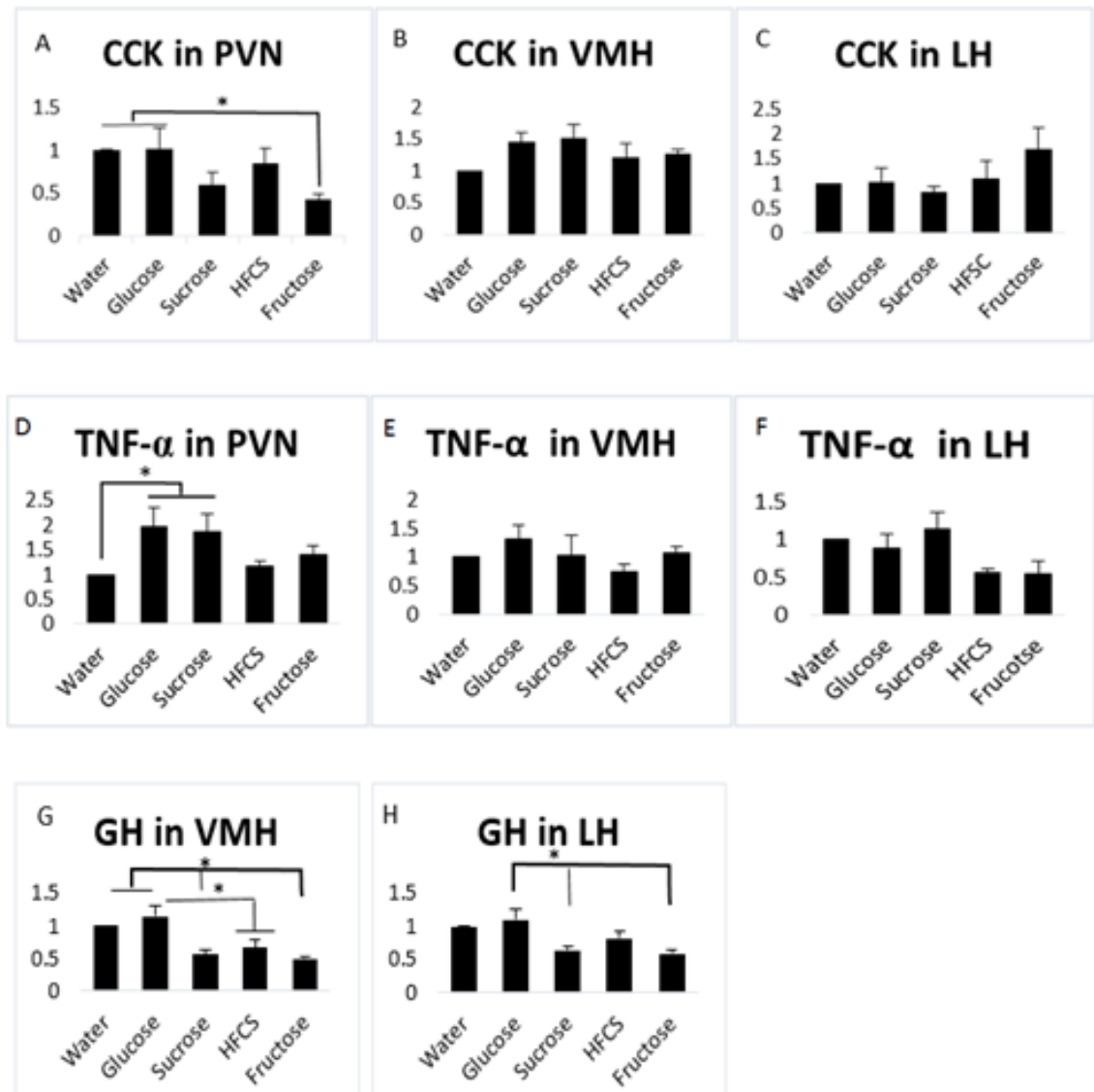

Figure 1. Different sugars affect the expression of CCK, TNF- $\alpha$ and GH in the PVN, VMH and LH. (A-C) Fructose downregulated CCK mRNA expression in the PVN $(p<0.05)$, but not in VMH or the LH (D-F) Glucose and sucrose intake significantly increased the mRNA expression of TNF- $\alpha$ in the PVN $(\mathrm{P}<0.05)$ but not in the VMH or LH. $(\mathrm{G}-\mathrm{H})$ In the VMH, sucrose, HFCS and fructose all decreased GH expression whereas only sucrose and fructose decreased GH expression at a significant level (Figure 1G). Sucrose and fructose decreased GH expression in both VMH and LH when compared with glucose

\subsubsection{RAMP3, TRH and CRH}

When compared to controls, none of the sugars tested had a significant effect on RAMP3, TRH or CRH messages (Figure 2). However, we did detect some differences of RAMP3 and TRH between sugar groups. Both HFCS and fructose significantly decreased RAMP3 mRNA expression compared with glucose in the VMH (Figure 2B). In the LH RAMP3 expression was only significantly reduced in the HFCS group when compared to the glucose group (Figure 2C). Both fructose and HFCS reduced TRH expression when compared with sucrose in the PVN (Figure 2D). No differences were found in the VMH or LH (Figure 2E and 2F). We failed to detect any significant difference in $\mathrm{CRH}$ in any regions tested (Figure 2G-I).

\subsection{Effect of Fructose or Glucose on CCK, TNF $\alpha$ and GH Expression}

Pearson correlation analyses revealed that CCK expression was regulated by fructose but not by glucose. A statistically significant inverse correlation between CCK expression in the PVN and fructose intake $(r=-0.36$, $\mathrm{p}<0.05$ ) was found (Figure 3A-B). No correlations between fructose or glucose intake and $\mathrm{TNF}-\alpha$ were statistically significant (Figure 3C-D). GH expression is inversely correlated with fructose intake $(\mathrm{r}=-0.44, \mathrm{p}=0.01)$ and positively correlated with glucose intake $(r=0.59$, $\mathrm{p}<0.01$ ) (Figure 3E-F). The correlations between fructose or glucose intakes with other neuropeptides in any of the three hypothalamic regions sampled failed to achieve statistical significance.

\section{Discussion}

Sugar induced obesity is well documented, but how sugars induce obesity is still not clear. Differences in sugar metabolism in the liver are not sufficient to explain how long term ad libitum sugar access can cause weight gain and/or even obesity. We hypothesize that sugars can affect the expression pattern of hypothalamic neuropeptides that may bias energy balance in the long term. To test this hypothesis, we gave rats brief access to different sugar solutions. Our results showed that under ad libitum conditions, fructose intakes were comparable (or even a little lower(see also[14])) to intakes of other sugars. This ad libitum method more closely mimics conditions that in humans lead to excess caloric intake and obesity. It should be noted that some popular sweetened drinks may have over $12 \%$ sugar content [15] and some juices even have higher sugar content [16]. 

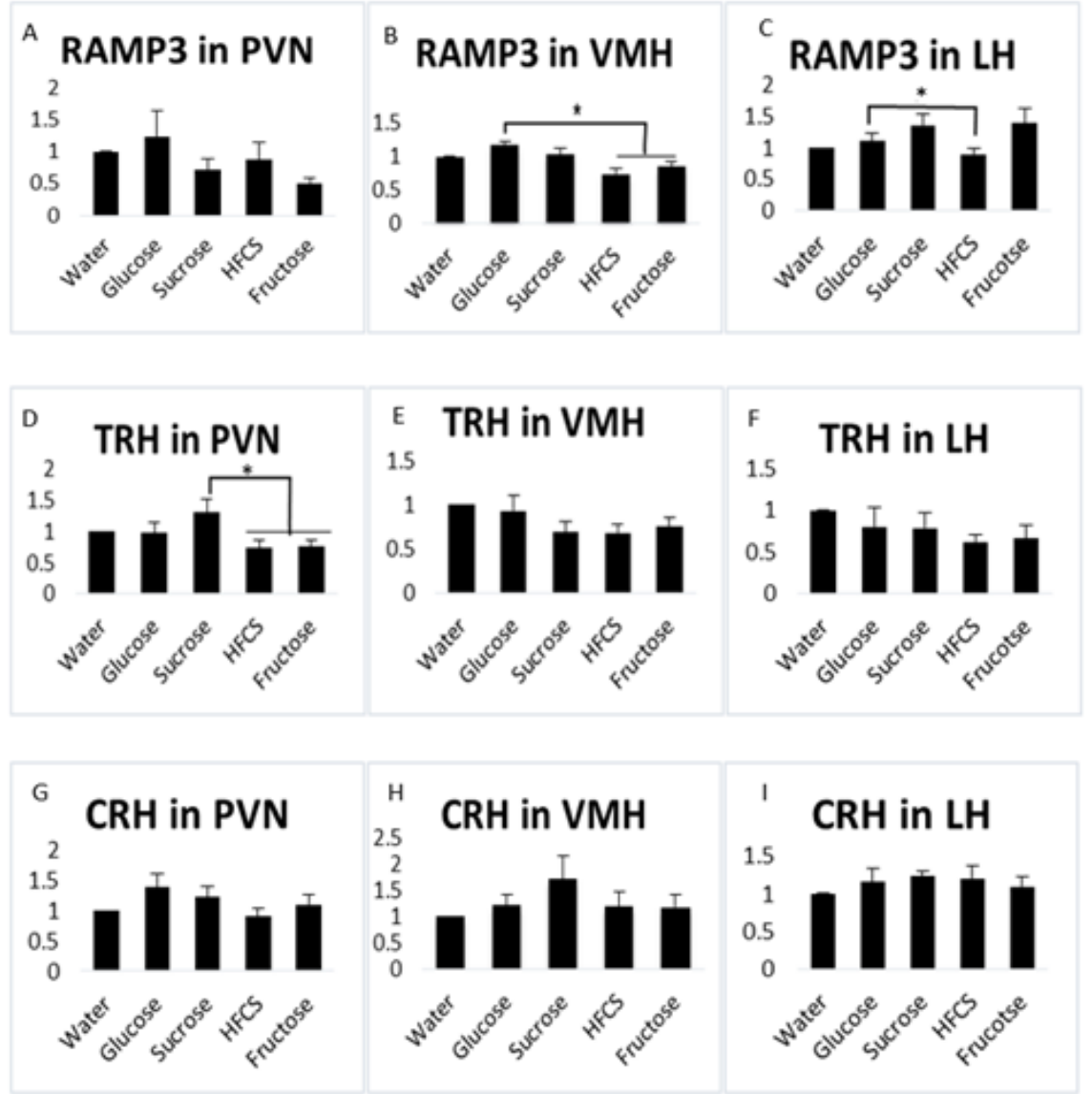

Figure 2. Different sugars affect the expression of RAMP3, TRH and CRH in the PVN, VMH and LH. (A-C) Both HFCS and fructose significantly decreased RAMP3 mRNA expression compared with glucose in the VMH. In the LH RAMP3 expression was only significantly reduced in the HFCS group when compared to the glucose group (D-F) Both fructose and HFCS reduced TRH mRNA expression when compared with sucrose in the PVN. No other differences were found in the VMH or LH. (G-I) No significant difference in CRH was found in the PVN, VMH or LH

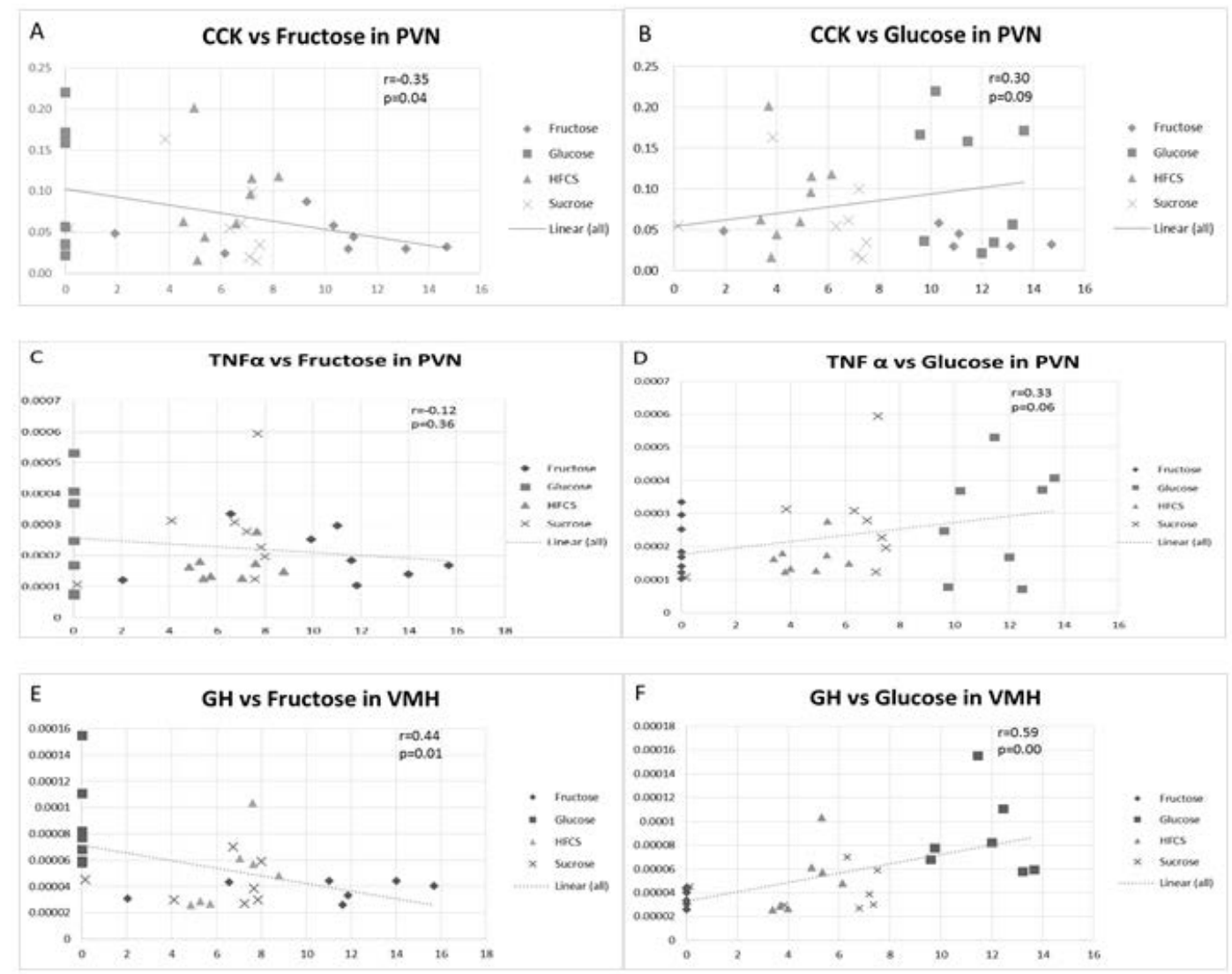

Figure 3. Effect of fructose or glucose on the expression of CCK, TNF- $\alpha$ and GH. (A-B) Expression of CCK mRNA in the PVN was inversely correlated with fructose intake but had no significant correlation with glucose intake. (C-D) TNF- $\alpha$ expression in the PVN was not significantly correlated with fructose or glucose intake. (E-F) GH mRNA expression in the VMH was significantly correlated with either fructose or glucose intake 
Free access to glucose, fructose, sucrose or HFCS can lead to excessive weight gain and/or obesity in the Sprague Dawley rats $[1,2,3]$. We found evidence that brief exposure to different sugar solutions can change the mRNA expression of CCK and TNF- $\alpha$ in the PVN as well as $\mathrm{GH}$ in the $\mathrm{VMH}$. This initial responses to sugar solutions are not the result from excess energy intake, as total caloric intake did not significantly differ among all five groups (Table 2). We also examined NPY and AgRP and failed to detect any significant differences (data not shown), which was consistent with our recent report [12].

CCK is well known as an effective hunger suppressant [17]. Brain CCK receptor deficiency results in hyperphagia and decreased responsiveness to high fat diet in rats [1820]. Dorsal medial hypothalamic CCK inhibits food intake for at least $22 \mathrm{~h}[21,22]$. CCK microinfused in the PVN inhibits gastric emptying and stimulates colonic transit in a dose dependent way [23]. It has been reported that CCK synthesis in the hypothalamus is disrupted during diabetes development [24]. CCK release from PVN in response to a gavaged meal is also compromised in obese ( $\mathrm{fa} / \mathrm{fa}$ ) Zucker rats [25]. These observations support the view that hypothalamic CCK plays an important role in energy control. We recently reported that 24 hour access to glucose solutions upregulated CCK expression whereas access to fructose resulted in significant downregulation of CCK [12]. In the current study, we replicated our finding that fructose access down-regulated CCK and can now report that this suppression takes place mainly in the PVN, not in the VMH or the LH. It has been previously reported that hypothalamic CCK is primarily expressed in PVN, typically in the parvocellular sub-nuclei [24].

TNF- $\alpha$ is an important chemokine involved in systemic inflammation. High doses of exogenous TNF- $\alpha$ can reduce food intake with weight loss being proportional to the decrease of both food and water intake [26]. It needs to be noted that the effect of TNF- $\alpha$ on food intake is mild compared with the effects of either insulin or leptin[27]. On the contrary, hypothalamic pro-inflammatory signaling can lead to impaired insulin sensitivity [28], increasing the potential for excess weight gain [29]. In the current study we found glucose and sucrose increased TNF- $\alpha$ mRNA expression. This effect is similar to that caused by high fat diet [30]. By contrast HFCS and fructose had no significant effect on TNF- $\alpha$ expression. The slight increase of TNF- $\alpha$ caused by glucose or sucrose was probably insufficient to have an effect on energy balance in the short term. Although fructose was reported to increase TNF- $\alpha$ in the whole hypothalamus [12], that observation was probably a reflection of the fact that the entire hypothalamus was sampled.

$\mathrm{GH}$ is known to be a peptide that contributes to the energy expenditure and lipid oxidation [31]. GH is critical for fat mobilization during fasting or starvation state when insulin is suppressed [32,33]. Adults with GH deficiency usually have increased body fat mass and decreased extracellular fluid volume [34]. We recently reported that $\mathrm{GH}$ expression was upregulated by glucose intake. In the current study we further found fructose decreased $\mathrm{GH}$ expression in specific hypothalamic areas [12]. Although the effect of glucose intake on the GH expression failed to reach statistical significance, the positive correlation between $\mathrm{GH}$ expression and glucose intake is robust $(\mathrm{P}<0.05)$. Because fructose can induce hypertriglyceridemia, our observation is also consistent with the fact that plasma triglycerides inhibit GH release [35].

RAMP3 can interact with several protein-coupled receptors. Adrenomedullin receptors and amylin receptors are two well-studied complex forms that require RAMP3. Adrenomedullin is a potent endogenous vasodilatory peptide. Increased amylin is associated with reduced body weight gain and adiposity [36]. Unlike CCK, glucose and fructose failed to affect the expression of RAMP3 in the PVN. Interestingly, although RAMP3 is mainly expressed in PVN, HFCS suppressed RAMP3 in both the VMH and the LH when compared to the glucose group, but not with the water-fed control group.

Finally, TRH is mainly located in the PVN and its secretion is connected with pituitary hormone release. TRH has an anorexigenic effect both by central or peripheral administration [37]. In contrast to the results from the whole hypothalamus reported earlier [12], fructose failed to significantly decrease TRH in the PVN, the VMH or the LH. It has been pointed out elsewhere [38] that TRH expression is highly specific to the PVN. We failed to replicate the previously reported finding (that fructose promoted decreases in TRH message and that HFCS intake downregulated CRH message). It is likely because that earlier work from our laboratory used tissues from a much larger region of the hypothalamus, whereas the sampling performed in this paper was confined to discreet hypothalamic nuclei or regions.

When we examined food intake and energy intake, we found that standard chow consumption was reduced but total energy intake remained unchanged. Although the reduction of CCK and GH or increase of TNF- $\alpha$ failed to affect energy intake immediately, the change in expression of these neuropeptides could possibly contribute to the obesity induced by long term use of sugars.

We have compared the effects of HFCS to the effects of other sugars including glucose, sucrose and fructose. Sucrose and HFCS have differing effects on several neuropeptides including TNF- $\alpha$, GH, RAMP3 and TRH. The consumption of sugars that contain more fructose than glucose (such as HFSC) can cause malabsorption[39]. As a result HFCS (55\% fructose, $41 \%$ glucose) has the potential to result in greater malabsorption than does sucrose, since sucrose is composed of $50 \%$ glucose and $50 \%$ fructose. Additionally, some sugars may also have unique effects on the gut microbiota. All these factors may lead to their distinct role in energy metabolism.

\section{Conclusions}

People who regularly consume sugar sweetened drinks usually have lower dietary quality as well as increased risks of metabolic syndrome [40]. For economic reasons, HFCS has replaced a great deal of the sucrose in our diets. We found a close link between the expression of the neuropeptide CCK and GH and fructose intake. No differences in CCK or GH between HFCS and sucrose groups were observed. We have presented evidence here that makes it clear that different sugars can and do have different effects on different targets, even within the hypothalamus. Our study clearly indicates that brief sugar intake from solution can change hypothalamic neuropeptides. This is a preliminary but requisite test to investigate the 
mechanism of sugar induced obesity. The mRNA measurement in the current study cannot predict protein levels but reflects on the expression changes of the neuropeptides which are critical for control of energy homeostasis. Further study is needed to confirm whether the change of these neuropeptides is sufficient for obesity induced by the long term use of sugars.

\section{Acknowledgments}

C. Z. is supported by the China Scholarship Council. This work was supported in part by a grant from the Maryland Agricultural Experiment Station to TWC.

\section{Conflict of Interest}

The authors declare no competing financial interests.

\section{References}

[1] Castonguay TW, Hirsch E, Collier G. Palatability of sugar solutions and dietary selection? Physiology \& Behavior 1981; 27: 7-12.

[2] Kanarek RB, Orthen-Gambill N. Differential effects of sucrose, fructose and glucose on carbohydrate-induced obesity in rats. The Journal of nutrition 1982; 112: 1546-54.

[3] Bocarsly ME, Powell ES, Avena NM, Hoebel BG. High-fructose corn syrup causes characteristics of obesity in rats: Increased body weight, body fat and triglyceride levels. Pharmacology Biochemistry and Behavior 2010; 97: 101-6.

[4] Schwartz MW, Woods SC, Porte D, Seeley RJ, Baskin DG. Central nervous system control of food intake. Nature 2000; 404: 661-71.

[5] Campbell ES, Castonguay TW. Fructose intake and circulating triglycerides: an examination of the roles of APOC 3 and FOXO1. The FASEB Journal 2013; 27: 1074.8.

[6] London E, Castonguay TW. High Fructose Diets Increase $11 \beta$ Hydroxysteroid Dehydrogenase Type 1 in Liver and Visceral Adipose in Rats Within 24 - h Exposure. Obesity 2011; 19: 92532.

[7] Masuzaki H, Flier J. Tissue-specific glucocorticoid reactivating enzyme, 11 beta-hydroxysteroid dehydrogenase type 1 (11 betaHSD1)--a promising drug target for the treatment of metabolic syndrome. Current drug targets. Immune, endocrine and metabolic disorders 2003; 3: 255-62.

[8] Masuzaki H, Paterson J, Shinyama H, Morton NM, Mullins JJ, Seckl JR, Flier JS. A transgenic model of visceral obesity and the metabolic syndrome. Science 2001; 294: 2166-70.

[9] Page KA, Chan O, Arora J, Belfort-DeAguiar R, Dzuira J, Roehmholdt B, Cline GW, Naik S, Sinha R, Constable RT. Effects of Fructose vs Glucose on Regional Cerebral Blood Flow in Brain Regions Involved With Appetite and Reward PathwaysFructose Consumption and Weight Gain. JAMA 2013; 309: 63-70.

[10] Stellar E. The physiology of motivation. Psychological review 1954; $61: 5$.

[11] Weingarten HP, Chang P, McDonald T. Comparison of the metabolic and behavioral disturbances following paraventricularand ventromedial-hypothalamic lesions. Brain research bulletin 1985; 14: 551-9.

[12] Colley DL, Castonguay TW. Effects of sugar solutions on hypothalamic appetite regulation (in press). Physiology \& Behavior 2014.

[13] Clément-Ziza M, Munnich A, Lyonnet S, Jaubert F, Besmond C. Stabilization of RNA during laser capture microdissection by performing experiments under argon atmosphere or using ethanol as a solvent in staining solutions. Rna 2008;14:2698-704.

[14] Lindqvist A, Baelemans A, Erlanson-Albertsson C. Effects of sucrose, glucose and fructose on peripheral and central appetite signals. Regulatory Peptides 2008; 150: 26-32.
[15] Ventura EE, Davis JN, Goran MI. Sugar content of popular sweetened beverages based on objective laboratory analysis: focus on fructose content. Obesity 2011; 19: 868-74.

[16] Walker RW, Dumke KA, Goran MI. Fructose content in popular beverages made with and without high-fructose corn syrup. Nutrition 2014; 30: 928-35.

[17] Kraly FS, Carty WJ, Resnick S, Smith GP. Effect of cholecystokinin on meal size and intermeal interval in the shamfeeding rat. Journal of comparative and physiological psychology 1978; 92: 697.

[18] Schwartz GJ, Whitney A, Skoglund C, Castonguay TW, Moran TH. Decreased responsiveness to dietary fat in Otsuka LongEvans Tokushima fatty rats lacking CCK-A receptors. American Journal of Physiology-Regulatory, Integrative and Comparative Physiology 1999; 277: R1144-R51.

[19] Chen H, Kent S, Morris MJ. Is the CCK2 receptor essential for normal regulation of body weight and adiposity? European Journal of Neuroscience 2006; 24: 1427-33.

[20] Weiland TJ, Voudouris NJ, Kent S. The role of $\mathrm{CCK}_{2}$ receptors in energy homeostasis: insights from the $\mathrm{CCK}_{2}$ receptor-deficient mouse. Physiology \& Behavior 2004; 82: 471-6.

[21] Chen J, Scott KA, Zhao Z, Moran TH, Bi S. Characterization of the feeding inhibition and neural activation produced by dorsomedial hypothalamic cholecystokinin administration. Neuroscience 2008; 152: 178-88.

[22] Zhu G, Yan J, Smith WW, Moran TH, Bi S. Roles of dorsomedial hypothalamic cholecystokinin signaling in the controls of meal patterns and glucose homeostasis. Physiology \& Behavior 2012; 105: 234-41.

[23] Tebbe J, Mönnikes H, Pluntke K, Bauer C, Arnold R. Cholecystokinin (CCK) microinfused into the paraventricular nucleus of the hypothalamus (PVN) inhibits gastric emptying and stimulates colonic motor activity in the conscious rat. Gastroenterology 1998; 114: A1184-A5.

[24] Abramov A, Kolesnik YM, Trzhetsinskii S, Orlovskii nM. Changes in the cholecystokinin-synthesizing system of the hypothalamus in experimental diabetes mellitus in rats. Neuroscience and behavioral physiology 1999; 29: 621-4.

[25] De Fanti BA, Backus RC, Hamilton JS, Gietzen DW, Horwitz BA. Lean $(\mathrm{Fa} / \mathrm{Fa})$ but not obese (fa/fa) Zucker rats release cholecystokinin at PVN after a gavaged meal. American Journal of Physiology-Endocrinology and Metabolism 1998; 275: E1-E5.

[26] Mahony S, Tisdale M. Induction of weight loss and metabolic alterations by human recombinant tumour necrosis factor. British journal of cancer 1988; 58: 345.

[27] Romanatto T, Cesquini M, Amaral ME, Roman ÉA, Moraes JC, Torsoni MA, Cruz-Neto AP, Velloso LA. TNF- $\alpha$ acts in the hypothalamus inhibiting food intake and increasing the respiratory quotient-effects on leptin and insulin signaling pathways. Peptides 2007; 28: 1050-8.

[28] De Souza CT, Araujo EP, Bordin S, Ashimine R, Zollner RL, Boschero AC, Saad MJ, Velloso LcA. Consumption of a fat-rich diet activates a proinflammatory response and induces insulin resistance in the hypothalamus. Endocrinology 2005; 146: 4192-9.

[29] Swaroop JJ, Rajarajeswari D, Naidu J. Association of TNF- $\alpha$ with insulin resistance in type 2 diabetes mellitus. The Indian journal of medical research 2012;135:127.

[30] Wang X, Ge A, Cheng M, Guo F, Zhao M, Zhou X, Liu L, Yang $\mathrm{N}$. Increased hypothalamic inflammation associated with the susceptibility to obesity in rats exposed to high-fat diet. Experimental diabetes research 2012; 2012.

[31] Jørgensen JOL, Pedersen SB, Børglum J, Møller N, Schmitz O, Christiansen JS, Richelsen B. Fuel metabolism, energy expenditure, and thyroid function in growth hormone-treated obese women: A double-blind placebo-controlled study. Metabolism 1994; 43: 872-7.

[32] Sakharova AA, Horowitz JF, Surya S, Goldenberg N, Harber MP Symons K, Barkan A. Role of growth hormone in regulating lipolysis, proteolysis, and hepatic glucose production during fasting. The Journal of Clinical Endocrinology \& Metabolism 2008; 93: 2755-9.

[33] Gahete MD, Córdoba-Chacón J, Luque RM, Kineman RD. The rise in growth hormone during starvation does not serve to maintain glucose levels or lean mass but is required for appropriate adipose tissue response in female mice. Endocrinology 2012; 154: 263-9.

[34] Rosen T, Bosaeus I, TöIli J, Lindstedt G, Bengtsson B-Å. Increased body fat mass and decreased extracellular fluid volume 
in adults with growth hormone deficiency. Clinical Endocrinology 1993; 38: 63-71.

[35] Coxam V, Davicco M-J, Barlet J-P. Effect of triglycerides on growth hormone (GH)-releasing factor-mediated GH secretion in newborn calves. Domestic animal endocrinology 1989; 6: 389-93.

[36] Lutz TA. Control of energy homeostasis by amylin. Cellular and molecular life sciences 2012; 69: 1947-65.

[37] Schuhler S, Warner A, Finney N, Bennett G, Ebling F, Brameld J. Thyrotrophin-Releasing Hormone Decreases Feeding and Increases Body Temperature, Activity and Oxygen Consumption in Siberian Hamsters. Journal of neuroendocrinology 2007; 19: 239-49.
[38] Fekete C, Légrádi G, Mihály E, Huang Q-H, Tatro JB, Rand WM, Emerson $\mathrm{CH}$, Lechan RM. $\alpha$-Melanocyte-stimulating hormone is contained in nerve terminals innervating thyrotropin-releasing hormone-synthesizing neurons in the hypothalamic paraventricular nucleus and prevents fasting-induced suppression of prothyrotropin-releasing hormone gene expression. The Journal of Neuroscience 2000; 20: 1550-8.

[39] Rumessen JJ. Fructose and related food carbohydrates: sources, intake, absorption, and clinical implications. Scandinavian journal of gastroenterology 1992; 27: 819-28.

[40] Bray G, Popkin B. Calorie - sweetened beverages and fructose: what have we learned 10 years later. Pediatric obesity 2013; 8: 242-8. 